\title{
Case report \\ Facial and ocular presentations of Carbimazole induced agranulocytosis: A case report Teoh $\mathrm{SH}^{1}$, Liyana $\mathrm{S}^{2}$
}

\begin{abstract}
Summary:
We report a case of 61-year-old lady who presented with signs and symptoms of acute dacryocystitis, preseptal and facial cellulitis secondary to carbimazole induced agranulocytosis. Her condition was complicated by sepsis whereby she was admitted for intravenous antibiotics, subcutaneous Filgrastim administration and surgical intervention.
\end{abstract}

Kevwords: agranulocytosis, carbimazole, ocular

\section{Introduction:}

Agranulocytosis literally means total absence of granulocytes and some studies defined it using calculated absolute neutrophil count (ANC) with different reference levels (ie: $\mathrm{ANC}<100,<200$ or $<500 /$ microlitre $)^{1-3}$. It is a rare condition with incidence rate ranging from one to five cases per million populations per year and commonly drug induced $^{1}$. Despite the rarity, the consequence is serious whereby patients may develop life threatening sepsis. The groups of medication commonly associated with drug induced agranulocytosis (DIA) are antibiotic, analgesic, anti-inflammatory, psychotropic, antiarrhythmic, cardiovascular medications, anticonvulsant, chemotherapy and anti-thyroid ${ }^{2}$.

\section{Case report:}

A 61-year-old Malay housewife, with underlying diabetes and hypertension presented to emergency department with complain of unresolved high grade fever for ten days associated with right eye and facial redness. Prior to that, she had two visits to private clinics and was discharged with antibiotics. On further questioning, she was started on carbimazole two months earlier for symptomatic hyperthyroidism at government clinic. On examination, she was febrile at 38 degree Celcius and septic looking but vital signs were stable and no signs of hyperthyroidism.
Her right periorbital and lacrimal sac area were erythematous, warmth and tender on palpation with the lesion extended to right maxillary region.

Urgent full blood count revealed leukopenia with white cell count of $0.5 \times 10^{9} /$ litre and calculated ANC was $18.5 /$ microlitre. She was diagnosed to have right preseptal and facial cellulitis with acute dacryocystitis and early lacrimal sac abscess secondary to carbimazole induced agranulocytosis complicated by neutropenic sepsis. Carbimazole was withheld and intravenous Piperacillin-tazobactam 4.5g QID started during admission. Concurrently, subcutaneous Filgrastim was also administered for three days.

Throughout ten days of admission, her general condition improved, ANC increased to 532/microlitre and she remained euthyroid. Both wound swab and blood culture result came back positive with Pseudomonas aeruginosa sensitive to Piperacillintazobactam. Despite recovering from sepsis, the local lesion worsened with copious pus discharge from lacrimal sac. She underwent incision and drainage of lacrimal sac abscess and wound debridement to relieve local infective nidus. Post-operatively, daily dressing was done and the wound was clean. However, secondary wound closure was slow due to underlying diabetes and patient was discharged after

1. Teoh Soo Huat, Medical Lecturer \& Family Physician, Advanced Medical and Dental Institute, Universiti Sains Malaysia, 13200 Kepala Batas, Penang, Malaysia

2. Liyana Salleh, Medical OfficerKlinik Kesihatan Bakar Arang, 08000 Sungai Petani, Kedah. email: ooloioyoaonoaoo@yahoo.com.my

Correspondence to: Teoh Soo Huat, Medical Lecturer \& Family Physician, Advanced Medical and Dental Institute, Universiti Sains Malaysia, 13200 Kepala Batas, Penang, Malaysia, email: sakpn2000@gmail.com 
total of one month admission and scheduled for skin grafting.

Two weeks after discharged, she underwent right lower eye lid wound debridement and full thickness skin graft (donor site from posterior auricular). The operation was uneventful and her wound was well healed. Her hyperthyroidism remained in remission without medication and follow up was continued in the respective health clinic.

\section{Discussion:}

Thionamides remained the mainstay medical treatment for hyperthyroidism in the last 70 years $^{3}$. The commonly prescribed medications in this group are carbimazole and propylthiouracil. The former is usually preferred due to longer duration of action, convenient once daily dosing and lower incident of side effects ${ }^{4}$. Despite the advantages, carbimazole was found to be one of the major contributors of DIA which usually developed within two months of consumption ${ }^{2,3}$. Some studies found that advancing age; particularly those more than 50 years and women were predictors of DIA which corresponded with the demography of our patient ${ }^{1}$.

Sheng et al. reported that common presentations of anti-thyroid induced agranulocytosis are fever and sore throat which were initially diagnosed as acute pharyngitis and tonsillitis ${ }^{3}$. The same study also found other less frequently seen presentations such as pneumonia and urinary tract infection. Of note, only one case report found relating facial cellulitis to carbimazole induced agranulocytosis and none was found for ocular presentation may suggest such presentations are rare and easily missed ${ }^{5}$.

The first step of managing DIA is withdrawing the offending medication and normalisation of ANC is expected to be around eight days for carbimazole ${ }^{1,2}$. Concurrently, treatment of underlying infection should also be administered ${ }^{1,3}$. Anti-pseudomonal antibiotic such as Piperacillin-tazobactam is chosen in DIA patients complicated with sepsis as Pseudomonas aeruginosa is the commonest causative pathogen ${ }^{3}$. Additionally, Filgrastim which is a granulocyte colony stimulating factor injected subcutaneously may also improve recovery time, shorten hospital stay and reduce antibiotic usage ${ }^{1-4}$. Effectiveness of monitoring white cell count and white cell differential has long been studied and at the point of time, it is not an evidence based practice ${ }^{1}$. Major guidelines recommended having baseline full blood count prior starting anti-thyroid medications and repeat as needed during febrile episode and symptoms suggestive of infection ${ }^{1,3}$. Importantly, attending doctors should be mindful of the atypical presentation of DIA such as non oral or pharyngeal symptoms. Educating patient to seek medical attention at the earliest sign and symptom of infection may also improve the outcome of DIA ${ }^{1,3}$.

\section{Conclusion:}

Carbimazole induced agranulocytosis is rare but fatal if the diagnosis is missed. High index of suspicion is required to detect uncommon presentation of DIA and proceed to blood investigation because there is no effective continuous monitoring at the moment. Patients' early presentation and doctors' early detection may improve overall outcome.

\section{The learning key points from this case are:}

- It serves as a reminder to primary care providers regarding anti-thyroid induced agranulocytosis which is a rare but lethal side effect.

- It alerts primary care providers the atypical presentation of agranulocytosis.

\section{Acknowledgement: None}

Conflict of interest: None

\section{References}

1. Coates TD. Drug-induced neutropenia and agranulocytosis. In: Newburger P, Rosmarin AG, editors. UpToDate. [Internet]. Waltham, MA: UpToDate 2017 [cited July 9, 2017]. Available from: https://www. uptodate.com/contents/drug-induced-neutropenia-andagranulocytosis

2. Andersohn F, Konzen C, Garbe E. Systematic review: Agranulocytosis induced by nonchemotherapy drugs. Ann Intern Med 2007;146(9):657-65.

3. Sheng WH, Hung CC, Chen YC, et al. Antithyroid-druginduced agranulocytosis complicated by life-threatening infections. $Q J$ Med 1999;92(8):455-61.

4. Ross DS. Graves' hyperthyroidism in nonpregnant adults: Overview of treatment. In: Cooper DS, Mulder JE, editors. UpToDate. [Internet]. Waltham, MA: UpToDate 2017 [cited August 15, 2017]. Available from: https:// www.uptodate.com/contents/graves-hyperthyroidismin-nonpregnant-adults-overview-of-treatment

5. del Giudice P, Cua E, Bernard E, et al. Pseudomonas aeruginosa ecthyma gangrenosum and facial cellulitis complicating carbimazole-induced agranulocytosis. Arch Dermatol 2006;142(12):1650-66. 